\begin{tabular}{|l|l|l|}
\hline M.T. & Mark T. & Silva $^{\mathrm{a}}$ \\
\hline R.S. & Robin S. & Howard $^{\mathrm{a}}$ \\
\hline L.D. & Luke D. & Kartsounis $^{\mathrm{b}}$ \\
\hline R.W. & Ralph W. & Ross-Russell $^{\mathrm{a}}$ \\
\hline
\end{tabular}

'Department of Neurology, St. Thomas' Hospital, and 'Department of Neuropsychology, The National Hospital for Neurology and Neurosurgery, London, UK

\title{
The Alien Grasp Reflex
}

\section{Dr. R.S. Howard, Department of Neurology, St. Thomas' Hospital, London SE1 7EH (UK)}

Following brain damage a number of primitive reflexes emerge including a group of prehensile movements, the most common being the grasp reflex [1,2]. We report a case in which a profound grasp reflex, of which the patient was consciously aware, formed the main presenting complaint.

A 56-year-old right-handed female presented with a 2-month history of functional impairment of the left hand. Her main complaint was of being 'unable to let go of things'. This was most apparent as an inability to release grip with her left hand on objects such as door

\section{$03=42 \pi 81$ OU2 $=807$ SCAN 23}

ti

KV 1,

$\mathrm{AS} \quad .41$

SL 8

GT 8

TP 18P

$152 \mathrm{~B} \mathrm{H} / \mathrm{SP}$

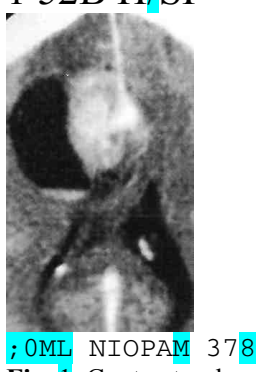

Fig. 1. Contrast-enhanced CT brain scan. There is a large ill-defined enhancing noncalcified cavitating mass lesion in the right frontal lobe, with enhancement of the medial border. Midline shift with spread of the mass across the fall $\mathrm{x}$ and egion is seen.

handles. Attempting to pull the hand away merely increased grip strength such that she would have to prize her fingers off the handle with the other hand. In other situations such as tying up rubbish bags, the left hand would tenaciously grip the bag and prevent release until the fingers were forcibly opened by the other hand. She had a flat affect with an expressionless face and a left-sided facial weakness. Limb tone wa increased on the left with lead-pipe rigidity, pyramidal weakness, brisk reflexes, an extensor plantar and a $4-6 \mathrm{~Hz}$ resting tremor. Bilateral grasp reflexes were present, the left being profound. Even with an examiner resting two static fingers in the grasping hand, the patient was unable to relax her grip and release the fingers to command. Attempting to withdraw the fingers from the patient's hand increased the grasp intensity. There were bilateral palmomental and foot grasp reflexes. Assessment on general intellectual ability (WAISS-R) and a range of focal cognitive tests revealed a verbal İQ of 82 and performance IQ 80 , reflecting a mild degree of general intellectual deterioration. Language, visual perceptual and memory skills were normal. Generation of words on fluency tests was adequate but she gave concrete interpretations to common proverbs. Her responses on a test of cognitive estimation were below normal limits. She had considerable difficulty with a Stroop task. Thus, the most notable feature of her neuropsychological assessment was her impaired performance on tests of frontal lobe executive function. A CT brain scan was obtained (fig. 1) and a diagnostic aspiration biopsy was performed. Histology of the lesion was that of an anaplastic oligodendroglioma. After surgery she received a radical course of radiotherapy receiving $60 \mathrm{cGy}$ in 30 fractions over 6 weeks. Following treatment her affect noticeably improved. Her tremor ceased but mild residual left-sided pyramidal weakness persisted. Although the primitive reflexes persisted, the left grasp was much less marked. Postoperatively the character of the grasp reflex had changed in that she was able to release the grip of her left hand voluntarily, exhibiting characteristics of a classical grasp reflex.

The presence of a grasp reflex, in full consciousness, indicates cerebral disease with damage characteristically occurring in eithe Short Reports

55

the frontal lobes or deep structures, especially the cingulate gyrus, supplementary motor area (SMA) and area 24 [2]. Damage of their projections to the basal ganglia may explain the initial parkinsonian tremor in this case [2]. Unlike the present case, most patients with forced grasping are able to release their grip when asked to do so [1,2]. Usually, in the absence of a change of stimulus after some seconds there is a gradual decline in the grasp reflex and if the object being gripped is static, then grip can be released [1]. We do not think that our patient's deficit was that of utilisation behavior as she was fully aware of the grasp gradual decline in the grasp reflex and if the object being gripped is static, then grip can be released [1]. We do not think that our patient's deficit was that of utilisation behavior as she was fully aware of the grasp activity and it occurred during a logical preconceived motor task [3]. Another possible analysis of her complaint may be made in the contex of the alien hand syndrome which is also associated with frontal lobe or
callosal lesions [4]. We do not consider our patient a typical case of alien hand as all other movements, apart from the grasp reflex itself, were perceived as normal. Thus, our patient manifested forced grasping of which she was fully aware, she could anticipate it and yet perceived it as alien. Grasp reflexes and alien hand syndromes are unlikely to be separate entities caused by different neuronatomical lesions probably being part of a spectrum of frontal lobe dysfunction. Accordingly for the present case we suggest the term 'alien grasp reflex'.

References

Seyffarth H, Denny-Brown D: The grasp reflex and the instinctive grasp reaction. Brain 1948;71:109-183.

De Renzi E, Barbieri C: The incidence of grasp reflex following hemispheric lesion and its relation to frontal damage. Brain 1992; 115:293-313.

Llhermitte F: 'Utilisation behaviour' and its relation to lesions of the frontal lobes. Brain 1983;106:237-255.

Bogen JE: The callosal syndrome; in Heilman KM, Valenstein E (eds): Clinics in Neuropsychology. New York, Oxford University Press, 1979, pp 308-359. 